\title{
Phase-Amplitude Coupling and Long-Range Phase Synchronization Reveal Frontotemporal Interactions during Visual Working Memory
}

\author{
Jonathan Daume, ${ }^{1}$ Thomas Gruber, ${ }^{2}$ Andreas K. Engel, ${ }^{1}$ and Uwe Friese ${ }^{1}$ \\ ${ }^{1}$ Department of Neurophysiology and Pathophysiology, University Medical Center Hamburg-Eppendorf, D-20246 Hamburg, Germany, and ${ }^{2}$ Institute of \\ Psychology, Osnabrück University, D-49069 Osnabrück, Germany
}

It has been suggested that cross-frequency phase-amplitude coupling $(\mathrm{PAC})$, particularly in temporal brain structures, serves as a neural mechanism for coordinated working memory storage. In this magnetoencephalography study, we show that during visual working memory maintenance, temporal cortex regions, which exhibit enhanced PAC, interact with prefrontal cortex via enhanced low-frequency phase synchronization. Healthy human participants were engaged in a visual delayed match-to-sample task with pictures of natural objects. During the delay period, we observed increased spectral power of beta $(20-28 \mathrm{~Hz})$ and gamma $(40-94 \mathrm{~Hz})$ bands as well as decreased power of theta/alpha band $(7-9 \mathrm{~Hz})$ oscillations in visual sensory areas. Enhanced PAC between the phases of theta/alpha and the amplitudes of beta oscillations was found in the left inferior temporal cortex (IT), an area known to be involved in visual object memory. Furthermore, the IT was functionally connected to the prefrontal cortex by increased low-frequency phase synchronization within the theta/alpha band. Together, these results point to a mechanism in which the combination of PAC and long-range phase synchronization subserves enhanced large-scale brain communication. They suggest that distant brain regions might coordinate their activity in the low-frequency range to engage local stimulus-related processing in higher frequencies via the combination of long-range, within-frequency phase synchronization and local cross-frequency PAC.

Key words: cross-frequency coupling; delayed match-to-sample; inferior temporal cortex; MEG; neural oscillations; subsequent memory effect

Significance Statement

Working memory maintenance, like other cognitive functions, requires the coordinated engagement of brain areas in local and large-scale networks. However, the mechanisms by which spatially distributed brain regions share and combine information remain primarily unknown. We show that the combination of long-range, low-frequency phase synchronization and local crossfrequency phase-amplitude coupling might serve as a mechanism to coordinate memory processes across distant brain areas. In this study, low-frequency phase synchronization between prefrontal and temporal cortex co-occurred with local cross-frequency phase-amplitude coupling to higher frequencies in the latter. By such means, ongoing working memory storage taking place in higher frequencies in temporal regions might be effectively coordinated by distant frontal brain regions through synchronized activity in the low-frequency range.

\section{Introduction}

Neural oscillatory activity in a wide range of frequencies has been found to be modulated during working memory (WM) as well

\footnotetext{
Received July 4, 2016; revised Oct. 7, 2016; accepted 0ct. 12, 2016.

Author contributions: J.D., T.G., A.K.E., and U.F. designed research; J.D. performed research; J.D. analyzed data; J.D. and U.F. wrote the paper.

This work was supported by grants from the German Research Foundation (FR-33661 to U.F. and T.G.;SFB 936/A3 to A.K.E.) and the European Union (ERC-2010-AdG-269716 to A.K.E.). We thank Tessa Rusch and Florian Göschl for helpful discussions on this manuscript, Guido Nolte and Arne Ewald for methodological support, and Christiane Reißmann and Nina Noverijan for assistance in data recording.

The authors declare no competing financial interests.
}

as long-term memory (LTM) processes in the human brain (Hanslmayr and Staudigl, 2014; Roux and Uhlhaas, 2014). Whereas beta $(13-30 \mathrm{~Hz})$ and gamma $(>40 \mathrm{~Hz})$ band activity are thought to play a crucial role in encoding, retrieval, and maintenance of stimulus material (Tallon-Baudry et al., 1998; Gruber et

Correspondence should be addressed to Jonathan Daume, Department of Neurophysiology and Pathophysiology, University Medical Center Hamburg-Eppendorf, Martinistrasse 52, D-20246 Hamburg, Germany. E-mail: j.daume@uke.de.

DOI:10.1523/JNEUROSCI.2130-16.2016

Copyright $\odot 2017$ the authors $\quad 0270-6474 / 17 / 370313-10 \$ 15.00 / 0$ 
al., 2004; Honkanen et al., 2015; Schneider and Rose, 2016), activity in lower frequencies of the theta $(4-7 \mathrm{~Hz})$ and alpha $(8-12$ $\mathrm{Hz}$ ) ranges was mainly associated with memory-guided topdown control of stimulus processing (Staudigl and Hanslmayr, 2013; Jensen et al., 2015).

In recent years, the interactions across frequency bands also became a topic of intense investigations. It has been suggested that the synchronization between oscillations from different frequency ranges serves as a possible mechanism for ongoing WM storage (Canolty and Knight, 2010; Roux and Uhlhaas, 2014). Especially, theta-gamma phase-amplitude coupling (PAC), in which the amplitude of gamma oscillations is coupled to the phase of theta oscillations, has been observed during memory maintenance in animals and humans (Tort et al., 2008; Axmacher et al., 2010). PAC, particularly observed in temporal lobe structures, is thought to reflect a neural correlate for WM storage (Fell and Axmacher, 2011).

Besides the temporal lobes, areas of the prefrontal cortex have been associated with WM processes. Instead of being directly involved in the storage of stimuli, however, recent theories have linked the PFC preferably with processes of WM monitoring and control (Sreenivasan et al., 2014). Presumably, prefrontal areas rather interact with sensory cortices to coordinate resources among areas involved in content processing and storage (Lara and Wallis, 2014). A mechanism suggested to guide such communication between distant brain regions is based on phase synchronization of neural oscillations within different frequency bands (Engel et al., 2001; Fries, 2015). In fact, increased phase synchronization between the PFC and temporal areas during WM processes especially in the theta/alpha frequency band has been demonstrated (for review, see Fell and Axmacher 2011).

Therefore, we hypothesize that areas that exhibit enhanced PAC during WM maintenance also concurrently communicate with other, presumably frontal brain regions through long-range phase synchronization. Such combination of long-range phase synchronization and local PAC might ensure efficient long-range integration and coordination of ongoing high-frequency WM processes taking place in distant brain areas. Some evidence for a functional relationship between PAC and long-range phase synchronization comes from a study in rats. von Nicolai et al. (2014) found that brain areas that exerted enhanced local theta-gamma PAC were further interconnected by enhanced phase synchronization within the theta band. Given the proposed role of frontal regions in monitoring WM storage in distant sensory areas, one could speculate that the control of frontal regions over highfrequency activity in sensory regions might be achieved through long-distant phase synchronization in low-frequency bands, locally combined with the coordination of high-frequency oscillations in sensory areas via cross-frequency PAC.

Thus, in the present study we investigated oscillatory activity during WM maintenance processes reflected by local PAC and longrange phase synchronization. We recorded oscillatory brain activity from healthy human participants using whole-brain magnetoencephalography (MEG) during a visual delayed match-to-sample task with pictures of natural objects. Our first goal was to observe local PAC in brain areas specifically involved in visual WM storage. To that end, we used a nonmnemonic control task that closely matched the perceptual and attentional demands of the visual WM task. Comparing memory-related activity to that of the control task, we expected to find enhanced PAC in brain regions known to be involved in the processing of visual sensory information. These areas were then assumed to exhibit enhanced low-frequency phase syn- chronization to remote areas within the visual WM network, in particular to the PFC. Such frontotemporal interactions could indicate top-down controlled coordination of WM storage processes expressed by local PAC.

\section{Materials and Methods}

\section{Participants}

Twenty-seven healthy volunteers (age, $24.6 \pm 2.7$ years; 14 female; all right-handed) received monetary compensation for their participation in the study ( $10 €$ per hour). All participants reported normal or corrected-to-normal vision and no history of psychiatric or neurological disorders. The study protocol was approved by the Institutional Review Board and was fully in line with the Declaration of Helsinki. Participants gave their informed written consent before the recording.

\section{Experimental procedure}

The experiment consisted of a WM and an unannounced subsequent recognition part. The WM part contained two conditions: (1) a visual delayed match-to-sample task (memory condition) and (2) a nonmnemonic visual discrimination task (control condition; Fig. 1A). A total of 420 line drawings depicting natural objects were presented. For these stimuli, we manually edited the picture set introduced by Snodgrass and Vanderwart (1980) by changing a variety of individual image features (e.g., adding, removing, rotating, or resizing object details). The resulting stimulus set consisted of two versions of each visual object that were mostly similar, yet clearly distinguishable. Importantly, these stimuli prevented any reliable verbalization strategies in view of the delayed match-to-sample task (see Fig. $1 A$ for an example stimulus pair).

Each trial of the WM condition started with a light gray fixation dot presented against a medium gray background in the middle of the screen. After an interval of 2500-3000 ms (randomly jittered in steps of $100 \mathrm{~ms}$ ), the sample stimulus, a dark gray line drawing of a natural object, substituted the fixation dot and was presented for $200 \mathrm{~ms}$. The fixation dot then reappeared for a period of $3000 \mathrm{~ms}$ (delay period), followed by a $200 \mathrm{~ms}$ presentation of the probe stimulus (Fig. 1A). The probe stimulus was either identical to the sample stimulus or a modified version of it. Participants were instructed to remember the sample stimulus for the duration of the delay period and to report whether the probe stimulus matched the sample stimulus or not by pressing one of two buttons with their right index or middle finger, respectively (response mapping was counterbalanced across participants). Participants received visual feedback about the correctness of their response.

The trial sequence of the control condition equaled that of the memory condition, except for the presentation of the probe stimuli. In the control condition, each trial included the presentation of an object picture (200 $\mathrm{ms}$ ) followed by the presentation of either a horizontally or vertically oriented ellipse (50 ms) after $3000 \mathrm{~ms}$ of fixation (Fig. 1A). Participants were instructed to report the orientation of the ellipse and were not required to memorize the sample stimulus. By adjusting luminance and shape of the ellipse dependent on the participants' performances in the memory condition on a trial-by-trial basis, we assured comparable performance levels in both conditions. We used eight different shapes per orientation ranging from circle-like (difficult) to egg-like (easy). To disguise the direct relationship between performance and ellipse appearance, the ellipse was generated with a random contrast and shape in $20 \%$ of the trials. To familiarize participants with the task, a short training session was conducted before the recording. In this training session, participants performed four trials for each of the two conditions. Training stimuli were not part of the subsequent recording sessions, in which participants completed five blocks per condition with 28 trials in each block. In total, 280 trials were shown, i.e., 140 trials per condition. All trials contained unique sample/probe stimuli pairs. Conditions were presented blockwise in an alternating order. Before each block, participants were informed which of the two tasks they would perform. After completion of the first part of the experiment, a 15 min break was scheduled, in which participants watched a short movie clip.

In the second part of the study, a recognition test was administered including all 140 objects of the WM condition (memory-old), all 140 objects of the control condition (control old), and 140 new objects (new). 


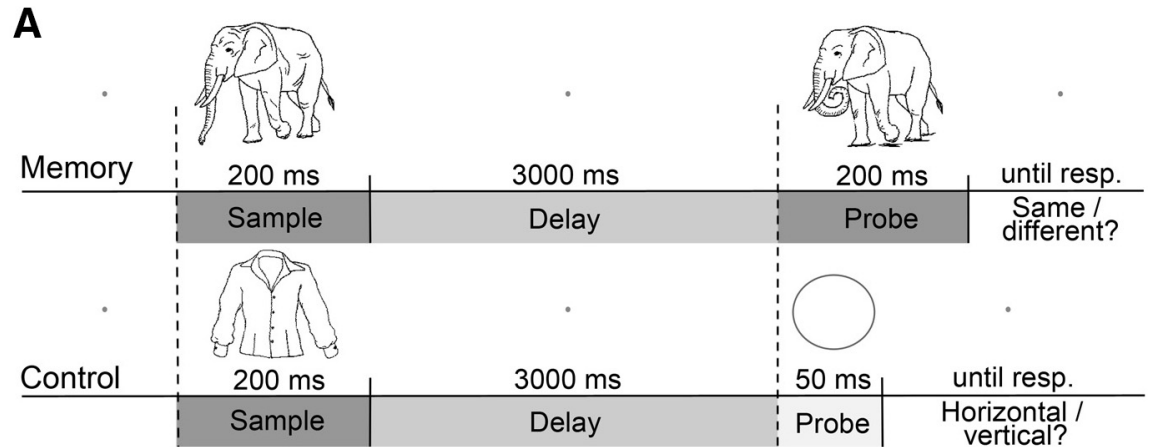

B

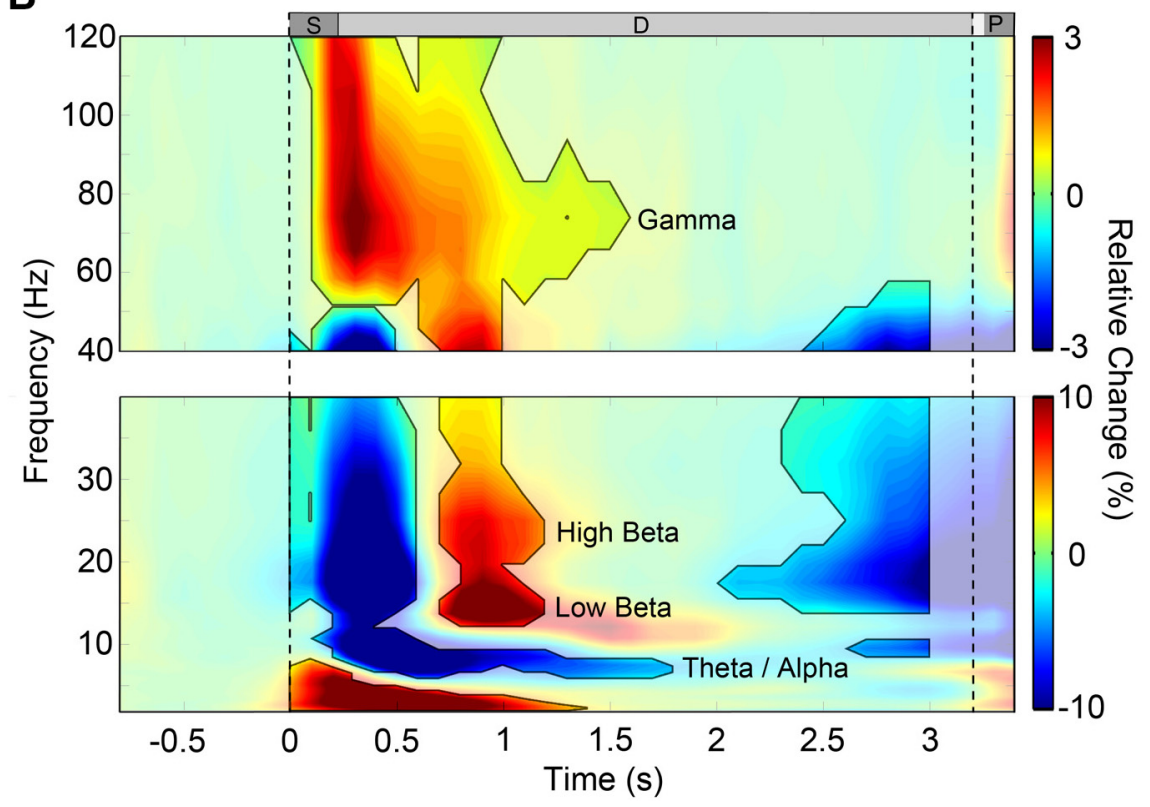

Figure 1. Schematic illustration of the trial sequence in the WM part and grand average of spectral power. $A$, In both conditions, a sample stimulus was presented for $200 \mathrm{~ms}$, followed by a $3000 \mathrm{~ms}$ delay period with no visual stimulation. In the memory condition, the sample stimulus had to be compared with a probe (also presented for $200 \mathrm{~ms}$ ) that could either be the same or a modified version of the sample stimulus. In the control condition, participants had to judge whether the probe stimulus (presented for $50 \mathrm{~ms}$ ) was either a horizontally or vertically oriented ellipse. $\boldsymbol{B}$, Time-frequency representation of spectral power throughout the entire trial averaged across all sensors, participants, and the two conditions shown separately for low and high frequencies. Dashed lines denote sample and probe stimulus onset and marked areas with intense colors represent significant changes relative to baseline (FDR corrected, $\alpha=0.01$ ). Warm colors depict increase, and cold colors indicate reduced power. Statistical tests were conducted for time points between 0 and $3000 \mathrm{~ms}$. Based on oscillatory activity within the delay window, we chose four frequency bands of interest for additional analysis: theta/alpha $(6.7-8.5 \mathrm{~Hz})$, low beta $(13.7-17.5 \mathrm{~Hz})$, high beta $(19.7-28.3 \mathrm{~Hz})$, and gamma (40.5-94.3 Hz). S, Sample stimulus; D, delay period; $P$, probe stimulus.

Participants were not aware of this administration until they finished the movie. Stimuli were presented for $200 \mathrm{~ms}$, and participants were asked to rate whether in relation to the first part of the experiment the presented object was "certainly old," "rather old," "rather new," or "certainly new" by pressing one of four buttons with their right hand. Visual feedback about the correctness of their answers was provided after each response. Participants performed 10 blocks with 42 trials per block. Stimulus assignments to the three conditions memory, control, and new were randomly shuffled for each participant.

Stimuli were presented using Matlab (version 8.0, R2012b, MathWorks; RRID: SCR_001622) and Psychtoolbox (version 3.0.10; Brainard, 1997; RRID: SCR_002881) on a Dell Precision T5500 with the Windows 7 Professional 64-bit operating system. The visual stimuli were projected onto a matte projection surface at $60 \mathrm{~Hz}$ with a resolution of $1280 \times 1024$ pixels positioned $65 \mathrm{~cm}$ in front of participants.

Data acquisition and preprocessing

We recorded MEG using a 275-channel whole-head system (Omega, CTF Systems) at a sampling rate of $1200 \mathrm{~Hz}$ in a dimly lit, sound- attenuated, and magnetically shielded room. Additional $\mathrm{Ag} / \mathrm{AgCl}$ electrodes were used to record eye, muscle, and cardiac activity. Head positions were tracked on-line during the recording and corrected between experimental blocks as soon as the movement exceeded 5 $\mathrm{mm}$. Seven channels were malfunctioning and therefore excluded from additional analysis.

Behavioral data were analyzed using $\mathrm{R}$ (version 3.1.1; R Developmental Core Team, 2014; RRID: SCR_001905) and RStudio (version 0.98.978; RStudio; RRID: SCR_000432). Reaction times deviating $2 \mathrm{SDs}$ from the mean of each participant were defined as outliers and removed from the behavioral analysis. Physiological data analysis was performed in Matlab 2013a using the M/EEG analysis toolbox FieldTrip (version 20140121; Oostenveld et al., 2011; RRID: SCR_004849) and custom-made scripts. First, the data were cut into epochs of $6.2 \mathrm{~s}$ including -1.5 to 4.7 s relative to the sample stimulus onset. Trials containing jumps and strong muscle artifacts were detected by semiautomatic procedures implemented in FieldTrip and were rejected after visual inspection. The remaining data were filtered with a high-pass filter at $0.5 \mathrm{~Hz}$; a low-pass filter at $170 \mathrm{~Hz}$; and three band-stop filters between 48.5 and 51.5, 98.5 and 101.5, and 148.5 and $151.5 \mathrm{~Hz}$; and down-sampled to 600 Hz. Afterward, independent component analysis was used to manually remove remaining eyemovement, cardiac, and muscle artifacts based on visual inspection of the component's time course, spectrum, and topography. On average, $16.5 \pm 5.2$ components (mean \pm SD) were rejected. Finally, all trials were visually inspected, and trials containing artifacts that were not detected by the previous steps were removed. On average, 269.2 \pm 9.1 trials of the total of 280 trials remained from the preprocessing.

\section{Data analysis}

Testing all possible instances of PAC and within-frequency phase coupling in source space is computationally challenging and results in a massive multiple-comparison problem and very low statistical power. In an attempt to balance the total number of statistical tests without introducing too many a priori assumptions, we restricted our data analysis to oscillatory activity during the memory delay interval and proceeded in five main steps. (1) First, we tested an average of spectral power across both conditions and all sensors against a baseline window in each frequency and time bin. Based on significantly modulated power during the delay period, we selected frequency bands of interest. We tested condition differences in time-resolved spectral power in these frequency bands at the sensor level and in source space. (2) To investigate the significance of WM maintenance for long-term storage, we examined spectral power differences within the WM delay period between subsequently remembered and subsequently forgotten stimuli. (3) Furthermore, we computed local PAC during the delay period in the memory and the control condition for combinations of low-frequency phase and high-frequency amplitude. Cluster permutation statistics were used to identify regions with significant PAC during memory maintenance compared with the control condition. (4) Then, we explored whether regions with local PAC interacted with more distant brain areas. We computed the imaginary part of coherency (Nolte et al., 2004) between voxels with significant PAC and all other voxels. (5) Finally, we 
A

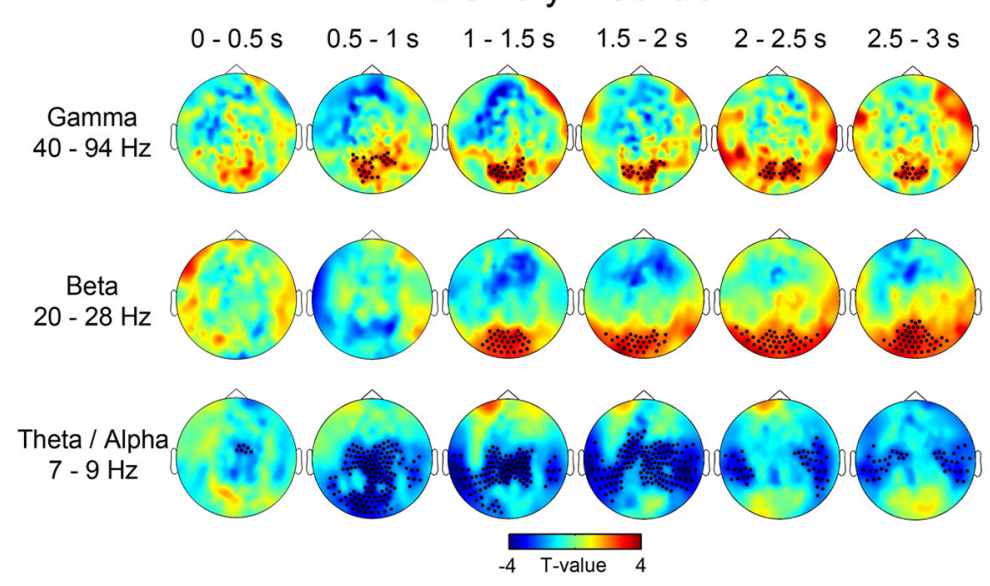

B Memory - Control
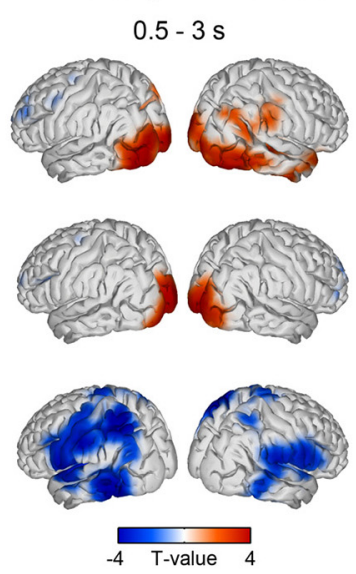

C
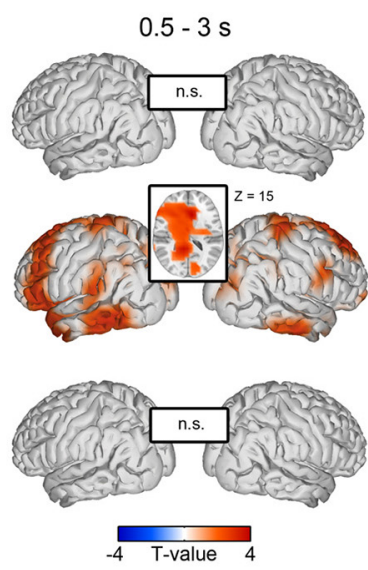

Figure 2. Spectral power differences in the frequency bands of interest. For reasons of clarity, results for the low beta band were omitted (see Results). $\boldsymbol{A}$, Clusters of significant power differences between the memory and the control conditions at the sensor level in steps of $500 \mathrm{~ms}$ ranging from 0 to $3000 \mathrm{~ms}$ for each frequency band of interest. Warm colors depict higher power in the memory, and cold colors indicate higher power in the control condition. $\boldsymbol{B}$, Cortical sources of the time-averaged differences between the memory and the control condition within the delay phase ranging from 500 to $3000 \mathrm{~ms}$ (FDR corrected, $\alpha=0.05$ ). The strongest effects were located in the left supramarginal gyrus, superior parietal lobe, and IT for theta/alpha frequencies; in bilateral striate cortices for beta frequencies; and in bilateral extrastriate areas for gamma frequencies. C, Differences in spectral power during WM maintenance between subsequently remembered and forgotten items (remembered - forgotten). Remembered items exhibited enhanced amplitudes in a large network of brain areas only in the beta band, with strongest increases in cingulate cortex and bilateral IT (FDR corrected, $\alpha=0.05$; $z$-coordinate is given in MNI space). No effects were found in the baseline window or in other frequency bands. SME, Subsequent memory effect; n.S., not significant.

assessed the directionality of information flow by computing the phaseslope index (PSI; Nolte et al., 2008) between areas showing significant effects for the imaginary part of coherency. Details for each analysis step are provided in the following section.

Spectral power. The recorded time series data at each sensor were transformed into time-frequency representations by wavelet convolution in the frequency domain. We used 35 complex Morlet wavelets spaced logarithmically from 2 to $120 \mathrm{~Hz}$ with a fixed number of seven cycles per wavelet. To obtain spectral estimates for induced power only, eventrelated fields were computed and subtracted from each sensor for each task and participant separately before the power analysis (Kalcher and Pfurtscheller, 1995). The wavelet convolution was performed for each trial separately and averaged within conditions. All trials (correct and incorrect) were taken into account. However, results for correct trials only were highly comparable with the results reported here. Power estimates for each frequency were baseline normalized using the average power of a prestimulus baseline window ranging from -800 to $-300 \mathrm{~ms}$ relative to sample stimulus onset.

We selected and averaged data within condition- and locationunspecific frequency bands that showed altered activity relative to baseline. For this purpose, we binned the data into steps of $100 \mathrm{~ms}$ and averaged it across conditions and sensors (Fig. 1B). We restricted our analyses to time windows before onset of the probe stimuli (3200 ms after sample stimulus onset). Consequently, we tested each time point from 0 $\mathrm{ms}$ (sample stimulus onset) to $3000 \mathrm{~ms}$ in all frequencies against the baseline (paired $t$ tests). We controlled for multiple comparisons by setting the false-discovery rate (FDR) correction to $\alpha=0.01$ (Storey, 2002). The main focus of the current study was on ongoing memory processes during the delay phase. Therefore, we grouped neighboring frequencies that showed related significant activity within the delay period into frequency bands of interest (see Results). We then tested power values between the conditions at each sensor and time window separately within the specified frequencies of interest. To this aim, we also averaged the data into windows of $500 \mathrm{~ms}$ and tested all windows between 0 and 3000 ms (Fig. 2A). To correct for multiple comparisons, we used the clusterbased randomization procedure as implemented in the FieldTrip toolbox (Maris and Oostenveld, 2007). In this procedure, for each time window, first a $t$ test is computed at every sensor separately. Neighboring sensors in terms of space and time falling below a $p$ value of 0.05 are grouped into clusters. Within each cluster, the sum of $t$ values is then computed. Cre- ating a null distribution through permutated data across participants (using 1000 permutations) and computing the maximum cluster-level test statistic results in a corrected $p$ value for each cluster. Clusters below an alpha level of $\alpha=0.05$ (two-sided) were considered significant.

At the cortical source level, spectral power differences between the conditions were estimated using dynamic imaging of coherent sources (DICS), a frequency-specific adaptive linear spatial filtering algorithm (DICS beamformer; Van Veen et al., 1997; Gross et al., 2001; Hipp et al., 2011). Estimates were computed in a 5003 voxel continuous grid based on individual MR images recorded from each participant. The grid was aligned to the 152 template brain [Montreal Neurological Institute (MNI); http://www.mcgill.ca]. Leadfields were calculated using the single-shell volume conductor model (Nolte, 2003). Cross-spectral density matrices were computed for the baseline and the delay window lasting from 500 to $3000 \mathrm{~ms}$. All matrices were weighted by the number of samples taken into account. We computed common filters by averaging the cross-spectral density matrices across all time windows and conditions. The three obtained orthogonal spatial filters were also combined into a single frequency-specific filter pointing in the direction of maximal variance and weighted with the first eigenvector's elements. To obtain power estimates in each voxel, the real-valued common filters were multiplied with the cross-spectral density matrix of each condition, frequency, and time window separately. Power values were averaged for the same frequency bands as on the sensor level. For each voxel and frequency band, we tested the baseline normalized power estimates between the conditions within the delay period and corrected for multiple comparisons by setting the FDR $\alpha=0.05$. Anatomical labeling of selected voxels in the space was performed using the NFRI functions, a set of Matlab functions described by Singh et al. (2005; http://www.jichi.ac.jp/brainlab/tools.html).

Subsequent memory effects. We first pooled data across confidence levels $($ remembered $=$ certainly old + rather old, forgotten $=$ certainly new + rather new). Next, trial numbers for the remembered and forgotten conditions were stratified to obtain comparable signal-to-noise ratios between conditions. Since there were fewer trials in the forgotten condition, we selected "remembered" trials that either closely preceded or followed a given "forgotten" trial. On average, $21.5 \pm 11$ trials were used for each participant.

Spectral power analysis was performed for two time windows, namely the baseline interval ( -800 to $-300 \mathrm{~ms}$ relative to sample stimulus on- 
A
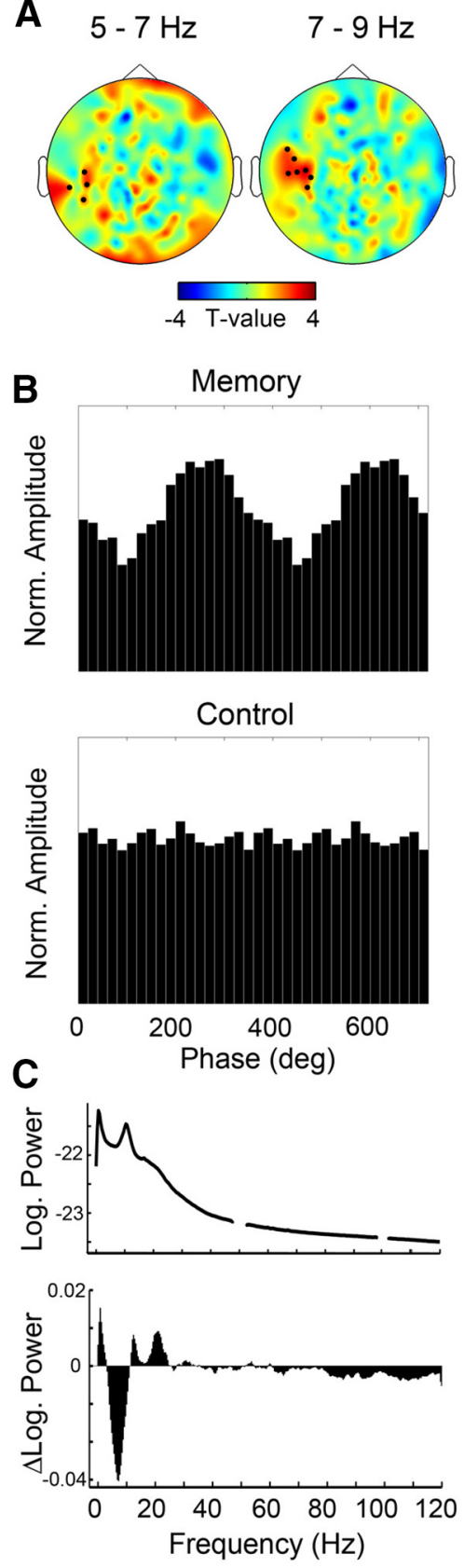

D

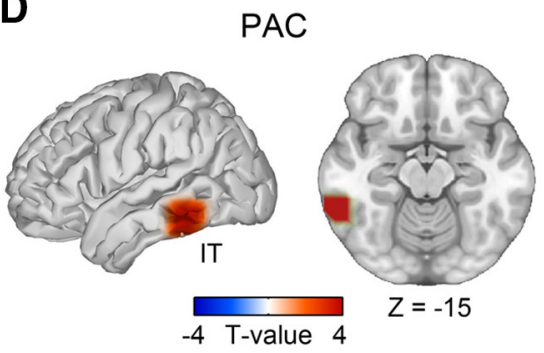

$\mathbf{E}$

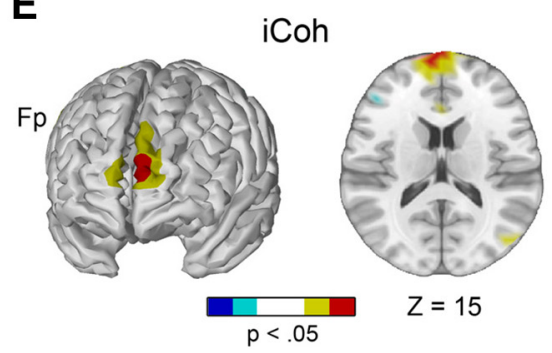

$\mathbf{F}$

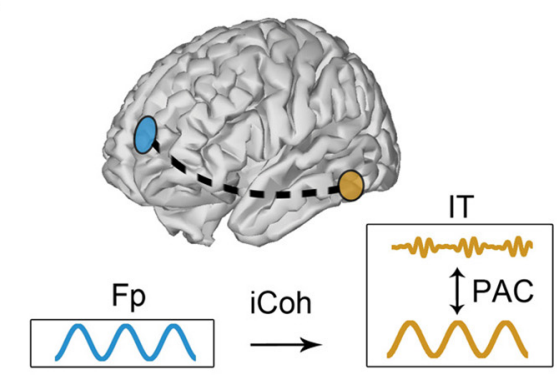

G

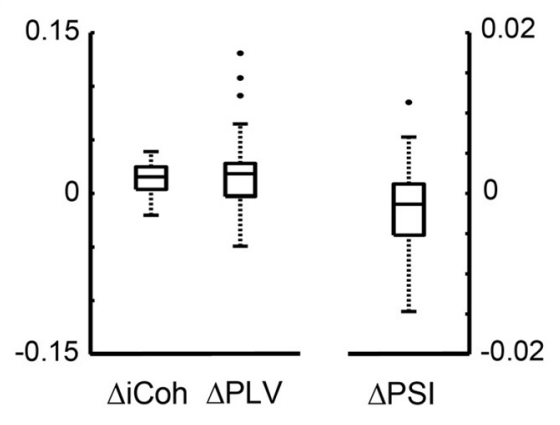

Figure 3. Local cross-frequency PAC and directed long-range, within-frequency phase synchronization. $A$, Result of the clusterbased permutation statistics on the PAC modulation indices (MIs). A cluster of left temporal sensors was apparent showing significantly increased Mls for phase frequencies in the theta/alpha and amplitude frequencies in the beta band $(20-28 \mathrm{~Hz})$ during memory maintenance compared with the control condition. No other clusters were found. $\boldsymbol{B}$, Beta amplitudes as a function of the theta/alpha phase within the sensors of the significant cluster from one participant. Only in the memory condition, the distribution of beta amplitudes across the theta/alpha phase differed from a uniform distribution. For illustrative purposes, the distributions are shown across two full cycles of the theta/alpha phase. C, Power spectrum during the delay period averaged across parietal, occipital, and temporal sensors and all participants. Top, Power averaged across both conditions; bottom, Their difference. This plot illustrates that power between the conditions differed in distinct narrowband frequency bands. If a rotation of the spectral slope underlies the differences in power between the conditions, then a consistent broadband decrease in low-frequency activity and an increase in high-frequency activity would be apparent. However, the observed differences in power between the conditions indicate that a change in power diversely affected specific narrowband oscillatory activity, allowing for a meaningful interpretation of interactions between frequency bands as measured by PAC. $D$, Significant differences in PAC at the source level were found in the left IT ( $z$-coordinate is given in MNI space). $E$, Computations of the imaginary part of coherency from the IT (seed voxel from PAC analysis) to all other voxels yielded significant phase synchronization to the left frontopolar cortex. Voxels that survived the correction for multiple comparisons are shown in red and dark blue colors (FDR corrected, $\alpha=0.05$ ), and voxels with uncorrected $p$ values smaller than 0.05 are shown in yellow and light blue colors (red/yellow, positive $t$ values; dark/light blue, negative $t$ values). $\boldsymbol{F}$, Schematic illustration of the combination of long-range, within-frequency phase synchronization and local crossfrequency PAC. The dashed line depicts the significant phase synchronization within the theta/alpha band between the left

set) and the delay period (500-3000 ms). Cross-spectral density matrices were computed as described above and projected to the 5003 voxel grid source space. Common beamformer filters were computed for both time windows separately. Frequencies were then averaged in the selected frequency bands of interest. Each frequency band and time window was tested for significant differences between the conditions in all voxels using paired sample $t$ tests. We corrected for multiple comparisons for each frequency band and time window separately (FDR $\alpha=0.05$ ).

Phase-amplitude coupling. We computed the modulation index (MI) as introduced by Tort et al. (2010) for a variety of frequency combinations. We estimated the phase of frequencies between 2 and $16 \mathrm{~Hz}$ (in steps of $2 \mathrm{~Hz}$; termed "phase frequencies") and amplitudes of frequencies between 18 and $120 \mathrm{~Hz}$ (in steps of $4 \mathrm{~Hz}$; termed "amplitude frequencies") and computed the MIs in both conditions for the time window between 500 and $3000 \mathrm{~ms}$ (delay period) of each trial. We filtered the time window of interest plus additional "buffer" windows of $200 \mathrm{~ms}$ before and after that window of interest for each sensor with a bandpass filter for the slow oscillation (bandwidth, $2 \mathrm{~Hz}$ ) and the fast oscillation (bandwidth, $4 \mathrm{~Hz}$ ). Using the Hilbert transformation, we extracted the instantaneous phase of the slow frequencies and the amplitude of the fast frequencies. To eliminate edge artifacts caused by filtering and applying the Hilbert transformation, the additional buffer windows were removed. All trials in each condition were then concatenated into one vector per sensor. By filtering, transforming, and cutting each trial separately, we prevented spurious PAC due to sharp edges in the data (Kramer et al., 2008). Thereafter, at each sensor separately, the MI for that pair of frequencies was computed as described by Tort et al. (2010). In brief, amplitudes of the high-frequency oscillation were assigned to each phase of the low-frequency signal. Then, low-frequency phases were binned into $20^{\circ}$ intervals (18 bins) and the mean amplitudes of the high-frequency oscillation in each bin were derived (see Fig. $3 B$ for an example). If no PAC is evident, the corresponding amplitude distributions across the bins are uniform. To test whether the empirical phase-amplitude distribution deviates from a uniform distribution, a discrete and normalized version of the Kullback-Leibler divergence, i.e., the MI, was calculated. This procedure was repeated for all combinations of phase and amplitude frequencies.

Statistical tests between the conditions were performed using the cluster-based randomization procedure as described above (see above,

frontopolar cortex and the left IT during WM maintenance as measured with the imaginary part of coherency. G, Distribution of iCoh, PLV, and PSI differences across participants. Data represent the differences of averaged connections in the IT and frontopolar cortex between the memory and the control condition. iCoh, Imaginary part of coherency; Fp, frontopolar cortex. 
Spectral power). The only difference was that here we used space, phase frequency, and amplitude frequency as cluster dimensions (as opposed to space and time in the spectral power analysis). We restricted statistical testing to amplitude frequencies from our frequency bands of interest, i.e., beta and gamma (frequencies from ranges lower than beta were used as phase frequencies). That is, cluster-based randomizations statistics were computed between the conditions for the amplitude frequency bands of interest, all considered phase frequencies, and all sensors.

To compute MIs at the source level, we first projected the data of each trial into source space. Since at this step the data were still in the time domain, we used linearly constrained minimum variance spatial filtering (Van Veen et al., 1997) for the projection. The calculations for PAC in source space were analogous to the sensor level analysis described above. As this analysis requires a vast amount of computational resources, we restricted the source analysis of PAC to a grid of 624 voxels and to the phase and amplitude frequencies showing significant differences in PAC in sensor space (see Results).

Source coherency analysis and phase-slope index. To reveal long-range connectivity between regions exhibiting local PAC and more distant brain areas, we computed coherency between pairs of voxels for the low-frequency band involved in PAC. As seed voxel, we used the coordinates of the PAC result in source space (see Results). First, we averaged the cross-spectral density matrices between 500 and $3000 \mathrm{~ms}$ in each condition and projected the result of each frequency bin within the theta/ alpha band to the 5003 voxel grid using linear beamforming (DICS; see above). For each frequency bin separately, coherency was computed from the seed voxel to all other voxels and averaged across all considered frequency bins. Because of the non-Gaussian distribution of coherency values, we applied Fisher's $Z$ transform to all coherency values. In the two-dimensional complex plane, the $Z$-transformed coherency values are approximately Gaussian, allowing for the utilization of parametric statistical tests (Nolte et al., 2004). To avoid spurious coupling attributable to volume conduction, we considered only the imaginary part of coherency, taking exclusively connections with non-zero phase lag into account (Nolte et al., 2004). This resulted in two coupling measures per voxel, one for the memory and one for the control condition. We statistically compared the conditions by computing two-sided, paired sample $t$ tests in each voxel and controlled for multiple comparisons by setting the FDR alpha level to $\alpha=0.05$.

It would be possible that a found connection between the region of interest and some other areas in the brain was just one of many, probably much stronger modulated connections existent in the data. Thus, to better evaluate and to reveal the significance of such a connection, we also computed the $t$ values for all other possible connections within a considered frequency band in a grid of 624 voxels.

Directionality of connections in source space was assessed by computing the PSI (Nolte et al., 2008), a measure that estimates the direction of information flow between two signals. The PSI was computed between voxels that showed a significant difference in the imaginary part of coherency comparing the memory and the control conditions. First, for each considered voxel pair, the complex coherency was calculated at each frequency bin within the frequency band of interest for both conditions separately (see above). Then, the phase-slope index was computed for each condition across all frequency bins within this frequency band as described by Nolte et al. (2008). A positive PSI indicates that the seed region acts as a sender, and negative values provide evidence for the seed region acting as a receiver. Differences between the memory and control conditions were evaluated with $t$ tests.

\section{Results}

\section{Behavioral results}

Data from one participant were rejected from analysis because of strong artifacts in the MEG recordings. Below, we report group performance as means complemented with SDs. Effect sizes are reported as Cohen's $d_{\mathrm{z}}$ for $t$ tests and partial $\eta^{2}$ for ANOVAs. Behavioral data from the remaining 26 participants showed no significant differences with respect to accuracy (Acc) or reaction times (RTs) between the memory condition (Acc, $79.0 \pm 5.2 \%$;
RT, $1064 \pm 227 \mathrm{~ms}$ ) and the control condition (Acc: 78.2 $\pm 5.9 \%$, $t_{(25)}=1.5, p=0.15, d_{\mathrm{z}}=0.3$; RTs: $1020 \pm 220 \mathrm{~ms}, t_{(25)}=1.6, p=$ $\left.0.13, d_{\mathrm{z}}=0.3\right)$.

Analysis of the behavioral data of the subsequent recognition task revealed that stimuli from the memory condition were better memorized than stimuli from the control condition. Participants remembered $83.5 \pm 10.6 \%$ from the memory condition, remembered $51.0 \pm 11.6 \%$ from the control condition, and correctly identified $66.6 \pm 11.1 \%$ objects as new stimuli. A one-way repeated-measures ANOVA revealed a significant main effect for the factor condition $\left(F_{(2,50)}=51.7, p<0.01, \eta_{\text {partial }}^{2}=0.7\right)$. Post hoc paired sample $t$ tests showed that accuracies for stimuli from the memory condition were significantly higher compared with stimuli from the control condition $\left(t_{(25)}=18.2, p<0.01, d_{\mathrm{z}}=\right.$ 3.6) as well as new stimuli $\left(t_{(25)}=5.0, p<0.01, d_{\mathrm{z}}=1.0\right)$. Accuracies for new stimuli were higher than for stimuli from the control condition $\left(t_{(25)}=3.9, p<0.01, d_{\mathrm{z}}=0.8\right)$. Signal detection theory (Green and Swets, 1966) sensitivity measure $d^{\prime}$ for the memory condition was $d^{\prime}=1.5 \pm 0.4$ and differed significantly from the control condition $d^{\prime}=0.5 \pm 0.2\left(t_{(25)}=16.2, p<0.01\right.$, $\left.d_{\mathrm{z}}=3.2\right)$. In addition, RTs showed a significant main effect for the factor condition $\left(F_{(2,50)}=24.1, p<0.01, \eta_{\text {partial }}^{2}=0.5\right)$. RTs for stimuli from the memory condition $(1071 \pm 172 \mathrm{~ms})$ were significantly shorter compared with the control condition $\left(1166 \pm 194 \mathrm{~ms} ; t_{(25)}=-5.5, p<0.01, d_{\mathrm{z}}=-1.1\right)$ and new stimuli $\left(1178 \pm 206 \mathrm{~ms} ; t_{(25)}=-4.9, p<0.01, d_{\mathrm{z}}=-1.1\right)$. RTs for stimuli from the control condition did not differ from those for new stimuli.

\section{Spectral power}

Numerous previous studies have associated WM as well as LTM processes with modulations in spectral power in a wide range of frequencies (Roux and Uhlhaas, 2014; for review, see Hanslmayr and Staudigl, 2014). Thus, we first analyzed spectral power differences between the memory and control conditions as well as between subsequently remembered and forgotten stimuli before the analysis of PAC and phase synchronization. Figure $1 B$ shows baseline corrected spectral power averaged across all MEG sensors, conditions, and subjects (grand average). As we were interested in neural activity only during WM maintenance, we restricted our analysis to frequencies showing significantly modulated amplitudes in the delay period (Fig. 1B, outlined areas). Hence, we analyzed four distinct frequency bands: theta/alpha $(6.7-8.5 \mathrm{~Hz})$, low beta $(13.7-17.5 \mathrm{~Hz})$, high beta $(19.7-28.3 \mathrm{~Hz})$, and gamma (40.5-94.3 Hz). Because of expanded temporal smearing of wavelets in the lower frequency range, we considered activity in the delta band $(\sim 2-5 \mathrm{~Hz})$ as representing early responses associated with the presentation of the sample stimulus, despite extending into the delay period. Thus, we did not include delta band frequencies in additional analyses. Modulated amplitudes shortly before the onset of the probe stimuli most likely represented attentional processes to the forthcoming stimuli and were therefore not considered further.

Figure 2 illustrates the differences in spectral power between the memory and the control conditions in the theta/alpha, high beta, and gamma frequency bands. Spectral power differences within the low beta band (data not shown) appeared to represent an average of the differences evident in the lower theta/alpha and the higher beta band and did not yield any information distinct from the neighboring frequency bands. Illustrations concerning low beta were therefore omitted for reasons of clarity, and all further results of the beta band refer to the $19.7-28.3 \mathrm{~Hz}$ range. 
Corrections for multiple comparisons, however, also included frequencies from the low beta band.

Frequencies in the theta/alpha band showed decreased amplitudes in a widespread cluster of mainly occipital and temporal sensors when participants were engaged in maintenance of to-bememorized objects during the WM task (Fig. 2A). Decreased amplitudes emerged mainly within 500 to $1000 \mathrm{~ms}$ after stimulus onset at a cluster of occipitoparietal and central sensors and propagated toward bilateral central and temporal sensors throughout the delay period. Oscillatory power analysis at the source level revealed decreased amplitudes in brain regions including higher visual processing areas such as middle temporal and parietal cortices. Lower amplitudes were also found in central and frontal areas such as somatosensory and inferior frontal regions (Fig. $2 B)$. The strongest decreases were located in the left supramarginal gyrus, superior parietal lobe, and inferior temporal cortex (IT).

Differences between the memory and the control conditions in the beta frequency band emerged at around $1000 \mathrm{~ms}$ after stimulus onset showing higher oscillatory activity in occipital sensors during the memory condition (Fig. 2A). This activity extended throughout the remaining delay period. The sources of the higher beta amplitudes in the WM condition were found in early visual areas with maxima being located in left and right primary visual cortices (V1; Fig. 2B).

Activity in the broadband gamma frequency range displayed enhanced amplitudes during the memory task compared with the control task in occipitoparietal sensors (Fig. 2A). Significantly increased activity emerged within 500 to $1000 \mathrm{~ms}$ after stimulus presentation and persisted throughout the entire delay period. Source estimates of the differences between the two conditions in the gamma range mainly revealed a bilateral involvement of early visual areas as well as higher visual areas in right inferior and medial temporal cortices and parietal regions (Fig. 2B). Maxima were located in bilateral extrastriate cortices (V2, V3) and left inferior occipitotemporal area.

To assess possible relationships between WM delay activity and LTM, we compared spectral power during maintenance of items that were successfully remembered in the subsequent recognition test with that of items subsequently forgotten. This was done for the memory condition only. Associations between WM maintenance and successful subsequent item recognition were specific to beta band activity during the delay interval. Higher spectral power for remembered stimuli compared with later forgotten stimuli was found in a widespread network of brain areas in temporal, frontal, and parietal cortices (Fig. 2C). The strongest increases in activity were located in the anterior and posterior parts of the cingulate cortex as well as bilateral IT. No effects were found in the baseline window or in other frequency bands.

\section{Phase-amplitude coupling}

First, we computed PAC for both conditions at all recorded sensors for different combinations of low- and high-frequency bands. Then, we used cluster-based permutation statistics to reveal clusters of sensors and frequencies showing significant differences in local PAC between the delay period of the memory and the control conditions. We found a cluster of left temporal sensors showing a significantly increased PAC during the WM task compared with the control condition $\left(p_{\text {corr }}<0.01\right.$; Fig. $\left.3 A\right)$ between the phase of frequencies at 5-7 and 7-9 Hz (center frequencies at 6 and $8 \mathrm{~Hz}$ ) and the amplitude of frequencies in the beta band. As an example for the different distributions of beta amplitude across the phases of theta/alpha, Figure $3 B$ depicts the average across all sensors from the significant PAC cluster separately for the memory and the control conditions from one representative participant.

The frequency bands involved in the PAC reported here also showed differences in spectral power between the conditions. This can be problematic as higher power values may lead to better phase estimations, which in turn may confound results on further phase synchronization measures. In our case, the IT showed enhanced theta/alpha-beta PAC but reduced spectral power in the theta/alpha band in the memory compared with the control condition (Figs. 2B, 3C). Therefore, differences in PAC between the conditions cannot be explained by confounded phase estimations in the lower phase-frequency band. Beta band power instead was enhanced for the memory condition. As we computed PAC as proposed by Tort et al. (2010), we normalized the spectral power in all amplitude frequencies before computing phase-amplitude correlations (see Materials and Methods). Hence, differences in PAC reported here cannot be explained by differences in spectral power of any of the involved frequency bands.

Moreover, given that the decrease of low-frequency activity in our data was accompanied by an increase of high-frequency activity in the memory compared with the control condition (Fig. 2 ), one could put into question whether indeed narrowband oscillatory activity, reflected by distinct peaks in the spectrum, caused the differences in spectral power between the two conditions (Aru et al., 2015). An alternative view on that would be that instead of changes in narrowband oscillations, rather a single manipulation of the underlying neural spiking activity caused correlated changes across the entire frequency spectrum (Voytek and Knight, 2015). An anticlockwise rotation of the spectral slope about a pivot frequency, for example, would lead to a broadband decrease in spectral power in low frequencies and an increase in power in high frequencies. Interpretations for functional roles of narrowband activity and especially the role for interactions between activity from different frequency bands (as quantified by PAC) would then, of course, be problematic since their change in activity would most probably not come from independent processes. To make sure that spectral power differences between the conditions were not caused by a rotation of the spectral slope, potentially leading to confounded PAC in the delay phase, we computed the power differences between the memory and the control conditions for all frequencies up to $120 \mathrm{~Hz}$ averaged across occipital, parietal, and temporal sensors in the delay period. Figure $3 C$ depicts the power spectrum averaged across both conditions (top) and their difference (bottom). Based on the fact that power was not consistently decreased in low frequencies and increased in high frequencies but showed distinct peaks in the difference spectrum, we can exclude the possibility that a single process (e.g., a manipulation of neural spiking statistics) caused a correlated change of spectral power across all frequencies. Instead, distinct peaks in the theta/alpha and beta bands indicated narrowband oscillatory activity and allowed for a meaningful interpretation of interacting frequency bands.

To further estimate the sources of the PAC effect we found on the sensor level, we also computed MIs for source space signals. We restricted the analysis to the stronger effect for $7-9 \mathrm{~Hz}$ phase frequencies and amplitude frequencies in the beta band and located the effect in the left IT (Fig. 3D).

\section{Long-range phase synchronization and directionality of information flow}

Having found PAC in the IT potentially reflecting a correlate of WM storage, we asked which brain regions might communicate 
with IT via low-frequency phase synchronization, e.g., to exert monitoring control. Hence, we examined whether the IT exhibited increased phase synchronization to any other region in the brain by computing the imaginary part of coherency from a seed voxel in the left IT to all other voxels within the theta/alpha band, which was involved in PAC. The results of the phase synchronization analyses are depicted in Figure $3 E-G$. After correcting for multiple comparisons, the left frontopolar cortex showed significantly increased phase synchronization with the left IT during WM maintenance compared with the nonmnemonic delay period of the control condition (Fig. $3 E, G$ ). This connection showed the strongest increase compared with the control condition among all possible connections within the theta/alpha band (see Materials and Methods).

Since the magnitude of coherency depends on the power of the signals, it is not a pure measure of phase synchronization. Hence, we retested the long-range connectivity effect using phase-locking values (PLVs), which are solely based on the phase information of the signals (Lachaux et al., 1999). Comparing the PLVs derived from the averaged signals in the IT and frontopolar cortex regions between the conditions revealed significantly stronger phase synchronization in the memory compared with the control condition $\left(t_{(25)}=2.9 ; p<0.01, d_{\mathrm{z}}=0.6\right.$; Fig. $\left.3 G\right)$.

Finally, we tested whether the direction of information flow would be compatible with the notion of frontopolar cortex exerting executive control over IT. Analysis of the PSI between IT and frontopolar cortex for frequencies in the theta/alpha band indicated that the frontopolar cortex served as the sender and the IT served as the receiver $\left(t_{(25)}=-1.83 ; p=0.04, d_{\mathrm{z}}=0.36\right.$; Fig. $\left.3 G\right)$.

\section{Discussion}

In this study, we found WM maintenance to be associated with higher sustained spectral power in the gamma $(40-94 \mathrm{~Hz})$ and beta $(20-28 \mathrm{~Hz})$ bands, whereas power in the theta/alpha band (7-9 Hz) was decreased. Higher beta power in widespread regions, including the IT, was predictive of successful subsequent LTM recognition. The IT also exhibited enhanced PAC between the phase of theta/alpha and the amplitude of beta oscillations during the memory delay period. Moreover, enhanced theta/ alpha phase synchronization between the IT and the frontopolar cortex revealed significant frontotemporal interactions during the maintenance of natural stimuli in visual WM.

\section{Sustained spectral power changes in gamma, beta, and theta/alpha bands}

In WM, enhanced gamma activity has been associated with ongoing processes reflecting the active maintenance of stimulus material (Roux and Uhlhaas, 2014). Our finding of enhanced gamma power during WM in visual ventral stream areas supports this functional role of gamma band activity.

Studies also showed enhanced beta amplitudes (Honkanen et al., 2015) or enhanced beta phase synchrony (Tallon-Baudry et al., 2001; Salazar et al., 2012) during WM maintenance. These and our results match the proposal that amplitude and/or synchrony of beta oscillations is amplified as long as a certain cognitive state is to be maintained (Engel and Fries, 2010).

It has been suggested that a decrease in alpha power reflects the release of relevant areas from inhibition, enabling them to be engaged in ongoing processes (Jensen and Mazaheri, 2010). Correspondingly, there is evidence for alpha amplitudes being suppressed in areas relevant to WM processing (Medendorp et al., 2007). Leszczyński et al. (2015) also showed suppressed alpha amplitudes in the hippocampus, which was related to WM con- tent maintenance. In accordance, our results demonstrate suppressed theta/alpha amplitudes in a broad network of visual sensory areas. Importantly, we also found strong amplitude decreases in the theta/alpha range in the IT, which seems to play a key role in WM and LTM processes.

Beta activity was higher for subsequently remembered compared with forgotten items during WM maintenance, with most pronounced differences in cingulate cortex and IT. Schneider and Rose (2016) demonstrated that beta amplitudes in the prestimulus period were enhanced during the intended encoding of visual stimulus material into LTM, indicating an important role for beta in LTM encoding. The posterior cingulate cortex (PCC) has been ascribed to diverse functional roles including memory and learning processes (Pearson et al., 2011; Heilbronner and Platt, 2013). Most prominently, it has been related to the socalled default mode network and internally directed cognition (Leech et al., 2012). This latter proposal and the association of PCC with memory processes are compatible with our finding of stronger activity in this region. Furthermore, we show that IT was involved in successful LTM encoding as well as in PAC during $\mathrm{WM}$, providing evidence for the suggested function of temporal regions as an interface for both WM and LTM processes (Fell and Axmacher, 2011). It also supports the view that the IT might serve as a key area for memory storage during the maintenance of natural objects in visual WM.

\section{Visual WM is associated with enhanced PAC in the IT}

Our finding of enhanced theta/alpha-beta coupling in the left IT strongly supports the view that PAC reflects a neural correlate of processes related to the reinstantiation of memory representations (Axmacher et al., 2010; Friese et al., 2013; Köster et al., 2014; Leszczyński et al., 2015; Alekseichuk et al., 2016; Heusser et al., 2016; Lega et al., 2016). Since we compared PAC during WM maintenance with that of a purely perceptional control task, we can exclude the possibility that PAC reflects more general, possibly memory-unspecific processing mechanisms (see also Park et al., 2013).

Here, we report PAC between frequencies of the theta/alpha and the beta bands whereas the majority of studies have so far described coupling between theta and gamma frequencies. On closer inspection, though, the borders of the frequency bands between which PAC occurs are not clear-cut. For instance, Axmacher et al. (2010) reported hippocampal theta-modulated amplitudes of oscillations within the $14-50 \mathrm{~Hz}$ beta/gamma range, which peaked at $28 \mathrm{~Hz}$. Thus, the modulated center-frequency rather was in the beta band. Moreover, their modulating frequencies ranged from 6 to $10 \mathrm{~Hz}$, which also includes our theta/alpha band. Therefore, the increase in theta/alpha-beta PAC during WM maintenance reported here in fact convincingly matches earlier findings.

Finding PAC in the left IT is consistent with previous evidence that this ventral stream region is involved in the WM processing of natural objects (Lueschow et al., 1994; Woloszyn and Sheinberg, 2009). A study by Woloszyn and Sheinberg (2009), for example, identified stimulus-selective cells in the monkey IT exhibiting modulated activity during WM. Given the proximity of IT to the medial temporal lobe (MTL) and the low spatial resolution of MEG source reconstructions, we cannot completely rule out that our results might reflect MTL activity to some degree. Note, however, that MTL structures and IT are likely to interact closely during WM maintenance. This has been demonstrated, for instance, by Axmacher et al. (2008), who found phase synchronization between the parahippocampal gyrus and IT 
using intracranial EEG recordings. In addition, Woloszyn and Sheinberg (2009) also speculated that the reinstatement of stimulus-specific IT activity might be governed by feedback projections from the PFC. Phase synchrony between the IT and the PFC, as described here, might reflect such a connection.

\section{IT and frontopolar cortex interact through low-frequency phase synchronization}

In recent years, the PFC has been preferably associated with processes of top-down control and monitoring rather than WM maintenance (Sreenivasan et al., 2014). It has been suggested that increased phase synchronization between the PFC and temporal areas during WM processes, especially in the theta/alpha frequency band, reflect such control processes (Fell and Axmacher, 2011). Our results support these ideas by demonstrating enhanced phase synchronization between the IT and the frontopolar cortex during WM maintenance. The frontopolar cortex found in our study is located somewhat anterior to the lateral prefrontal regions predominantly reported in WM studies. However, in line with our results, it has been linked to WM-related goal monitoring and attentional control processes (Braver and Bongiolatti, 2002; Ramnani and Owen, 2004). Recently, a study by Ryals et al. (2016) demonstrated a causal link between the frontopolar cortex and memory monitoring using theta-burst transcranial magnetic stimulation. They showed that stimulation of the frontopolar cortex causally influenced different measures of memory awareness, providing direct evidence of the involvement of the frontopolar cortex in memory monitoring. With evidence for an increased flow of information from frontopolar cortex to IT during WM maintenance, our study supports the idea that the frontopolar cortex might interact with temporal regions in a top-down manner to coordinate ongoing maintenance of visual object representations.

\section{Co-occurrence of local PAC and long-range phase synchronization as an efficient mechanism for top-down controlled WM maintenance}

Our results support the idea that the combination of within- and between-frequency coupling might serve as an efficient form of long-range communication between distant brain areas (von Nicolai et al., 2014; Hyafil et al., 2015). It has been suggested that phase synchronization in lower frequencies is better suited to establish interactions over wide distances, while in higher frequencies it is locally restricted (von Stein and Sarnthein, 2000). Following this idea, one could speculate that a combination of long-range, within-frequency phase synchronization and local cross-frequency PAC during WM maintenance serves as a mechanism of top-down coordination over long distances between frontal and sensory areas (Fig. $3 F$ ). In that sense, long-range interaction might be established through synchronized activity in lower frequencies between distant brain areas, combined with a local coupling to amplitudes of higher frequencies. Hence, instead of establishing spatially restricted synchronization to areas directly within high-frequency bands, distant brain areas could rather indirectly coordinate high-frequency activity through the combination of long-distance, low-frequency phase synchronization and local cross-frequency PAC. To the best of our knowledge, our study is the first to report direct evidence for such a mechanism during visual WM maintenance.

\section{Conclusions}

Our results point to a mechanism, in which the combination of local PAC and long-range phase synchronization might reflect long-range communication between distant brain areas. They suggest that during WM maintenance, the co-occurrence of both coupling types establish frontotemporal interactions between PFC and IT. As a result, a PFC-induced monitoring of highfrequency memory processes in the IT could be achieved by the phase synchronization of low-frequency activity between the areas, combined with the local coordination of high-frequency activity within the IT via PAC. Moreover, in all our analyses, we found the left IT to be among the brain areas showing the strongest task-relevant modulations: (1) spectral power in the theta/ alpha band was reduced in the left IT; 2 ) beta band power in the IT was predictive of successful long-term memory encoding; (3) the left IT exhibited WM-related PAC between theta/alpha and beta oscillations; and (4) theta/alpha phase synchronization was enhanced between left IT and frontopolar cortex with evidence for enhanced information flow from PFC to IT during memory maintenance.

\section{References}

Alekseichuk I, Turi Z, Amador de Lara G, Antal A, Paulus W (2016) Spatial working memory in humans depends on theta and high gamma synchronization in the prefrontal cortex. Curr Biol 26:1513-1521. CrossRef Medline

Aru J, Aru J, Priesemann V, Wibral M, Lana L, Pipa G, Singer W, Vicente R (2015) Untangling cross-frequency coupling in neuroscience. Curr Opin Neurobiol 31:51-61. CrossRef Medline

Axmacher N, Schmitz DP, Wagner T, Elger CE, Fell J (2008) Interactions between medial temporal lobe, prefrontal cortex, and inferior temporal regions during visual working memory: a combined intracranial EEG and functional magnetic resonance imaging study. J Neurosci 28:7304-7312. CrossRef Medline

Axmacher N, Henseler MM, Jensen O, Weinreich I, Elger CE, Fell J (2010) Cross-frequency coupling supports multi-item working memory in the human hippocampus. Proc Natl Acad Sci U S A 107:32283233. CrossRef Medline

Brainard DH (1997) The psychophysics toolbox. Spat Vis 10:433-436. CrossRef Medline

Braver TS, Bongiolatti SR (2002) The role of frontopolar cortex in subgoal processing during working memory. Neuroimage 15:523-536. CrossRef Medline

Canolty RT, Knight RT (2010) The functional role of cross-frequency coupling. Trends Cogn Sci 14:506-515. CrossRef Medline

Engel AK, Fries P (2010) Beta-band oscillations-signalling the status quo? Curr Opin Neurobiol 20:156-165. CrossRef Medline

Engel AK, Fries P, Singer W (2001) Dynamic predictions: oscillations and synchrony in top-down processing. Nat Rev Neurosci 2:704-716. CrossRef Medline

Fell J, Axmacher N (2011) The role of phase synchronization in memory processes. Nat Rev Neurosci 12:105-118. CrossRef Medline

Fries P (2015) Rhythms for cognition: communication through coherence. Neuron 88:220-235. CrossRef Medline

Friese U, Köster M, Hassler U, Martens U, Trujillo-Barreto N, Gruber T (2013) Successful memory encoding is associated with increased crossfrequency coupling between frontal theta and posterior gamma oscillations in human scalp-recorded EEG. Neuroimage 66:642-647. CrossRef Medline

Green DM, Swets JA (1966) Signal detection theory and psychophysics. New York: Wiley.

Gross J, Kujala J, Hamalainen M, Timmermann L, Schnitzler A, Salmelin R (2001) Dynamic imaging of coherent sources: studying neural interactions in the human brain. Proc Natl Acad Sci U S A 98:694-699. CrossRef Medline

Gruber T, Tsivilis D, Montaldi D, Müller MM (2004) Induced gamma band responses: an early marker of memory encoding and retrieval. Neuroreport 15:1837-1841. CrossRef Medline

Hanslmayr S, Staudigl T (2014) How brain oscillations form memories-a processing based perspective on oscillatory subsequent memory effects. Neuroimage 85:648-655. CrossRef Medline

Heilbronner SR, Platt ML (2013) Causal evidence of performance monitor- 
ing by neurons in posterior cingulate cortex during learning. Neuron 80:1384-1391. CrossRef Medline

Heusser AC, Poeppel D, Ezzyat Y, Davachi L (2016) Episodic sequence memory is supported by a theta-gamma phase code. Nat Neurosci 19: 1374-1380. CrossRef Medline

Hipp JF, Engel AK, Siegel M (2011) Oscillatory synchronization in large-scale cortical networks predicts perception. Neuron 69:387-396. CrossRef Medline

Honkanen R, Rouhinen S, Wang SH, Palva JM, Palva S (2015) Gamma oscillations underlie the maintenance of feature-specific information and the contents of visual working memory. Cereb Cortex 25:3788-3801. CrossRef Medline

Hyafil A, Giraud AL, Fontolan L, Gutkin B (2015) Neural cross-frequency coupling: connecting architectures, mechanisms, and functions. Trends Neurosci 38:725-740. CrossRef Medline

Jensen O, Mazaheri A (2010) Shaping functional architecture by oscillatory alpha activity: gating by inhibition. Front Hum Neurosci 4:186. CrossRef Medline

Jensen O, Bonnefond M, Marshall TR, Tiesinga P (2015) Oscillatory mechanisms of feedforward and feedback visual processing. Trends Neurosci 38:192-194. CrossRef Medline

Kalcher J, Pfurtscheller G (1995) Discrimination between phase-locked and non-phase-locked event-related EEG activity. Electroencephalogr Clin Neurophysiol 94:381-384. CrossRef Medline

Köster M, Friese U, Schöne B, Trujillo-Barreto N, Gruber T (2014) Thetagamma coupling during episodic retrieval in the human EEG. Brain Res 1577:57-68. CrossRef Medline

Kramer MA, Tort AB, Kopell NJ (2008) Sharp edge artifacts and spurious coupling in EEG frequency comodulation measures. J Neurosci Methods 170:352-357. CrossRef Medline

Lachaux JP, Rodriguez E, Martinerie J, Varela FJ (1999) Measuring phase synchrony in brain signals. Hum Brain Mapp 8:194-208. CrossRef Medline

Lara AH, Wallis JD (2014) Executive control processes underlying multiitem working memory. Nat Neurosci 17:876-883. CrossRef Medline

Leech R, Braga R, Sharp DJ (2012) Echoes of the brain within the posterior cingulate cortex. J Neurosci 32:215-222. CrossRef Medline

Lega B, Burke J, Jacobs J, Kahana MJ (2016) Slow-theta-to-gamma phaseamplitude coupling in human hippocampus supports the formation of new episodic memories. Cereb Cortex 26:268-278. CrossRef Medline

Leszczyński M, Fell J, Axmacher N (2015) Rhythmic working memory activation in the human hippocampus. Cell Rep 13:1272-1282. CrossRef Medline

Lueschow A, Miller EK, Desimone R (1994) Inferior temporal mechanisms for invariant object recognition. Cereb Cortex 4:523-531. CrossRef Medline

Maris E, Oostenveld R (2007) Nonparametric statistical testing of EEG- and MEG-data. J Neurosci Methods 164:177-190. CrossRef Medline

Medendorp WP, Kramer GF, Jensen O, Oostenveld R, Schoffelen JM, Fries P (2007) Oscillatory activity in human parietal and occipital cortex shows hemispheric lateralization and memory effects in a delayed double-step saccade task. Cereb Cortex 17:2364-2374. CrossRef Medline

Nolte G (2003) The magnetic lead field theorem in the quasi-static approximation and its use for magnetoencephalography forward calculation in realistic volume conductors. Phys Med Biol 48:3637-3652. CrossRef Medline

Nolte G, Bai O, Wheaton L, Mari Z, Vorbach S, Hallett M (2004) Identifying true brain interaction from EEG data using the imaginary part of coherency. Clin Neurophysiol 115:2292-2307. CrossRef Medline

Nolte G, Ziehe A, Nikulin VV, Schlögl A, Krämer N, Brismar T, Müller KR (2008) Robustly estimating the flow direction of information in complex physical systems. Phys Rev Lett 100:234101. CrossRef Medline

Oostenveld R, Fries P, Maris E, Schoffelen J-M (2011) FieldTrip: open source software for advanced analysis of MEG, EEG, and invasive electrophysiological data. Comput Intell Neurosci 2011:156869. CrossRef Medline

Park JY, Jhung K, Lee J, An SK (2013) Theta-gamma coupling during a working memory task as compared to a simple vigilance task. Neurosci Lett 532:39-43. CrossRef Medline

Pearson JM, Heilbronner SR, Barack DL, Hayden BY, Platt ML (2011) Posterior cingulate cortex: adapting behavior to a changing world. Trends Cogn Sci 15:143-151. CrossRef Medline

R Developmental Core Team (2014) R: a language and environment for statistical computing. R Foundation for Statistical Computing, Vienna. ISBN 3-900051-07-0.

Ramnani N, Owen AM (2004) Anterior prefrontal cortex: insights into function from anatomy and neuroimaging. Nat Rev Neurosci 5:184-194. CrossRef Medline

Roux F, Uhlhaas PJ (2014) Working memory and neural oscillations: alpha-gamma versus theta-gamma codes for distinct WM information? Trends Cogn Sci 18:16-25. CrossRef Medline

Ryals AJ, Rogers LM, Gross EZ, Polnaszek KL, Voss JL (2016) Associative recognition memory awareness improved by theta-burst stimulation of frontopolar cortex. Cereb Cortex 26:1200-1210. CrossRef Medline

Salazar RF, Dotson NM, Bressler SL, Gray CM (2012) Content-specific fronto-parietal synchronization during visual working memory. Science 338:1097-1100. CrossRef

Schneider SL, Rose M (2016) Intention to encode boosts memory-related prestimulus EEG beta power. Neuroimage 125:978-987. CrossRef Medline

Singh AK, Okamoto M, Dan H, Jurcak V, Dan I (2005) Spatial registration of multichannel multi-subject fNIRS data to space without MRI. Neuroimage 27:842-851. CrossRef Medline

Snodgrass JG, Vanderwart M (1980) A standardized set of 260 pictures: norms for name agreement, image agreement, familiarity, and visual complexity. J Exp Psychol Hum Learn Mem 6:174-215. CrossRef

Sreenivasan KK, Curtis CE, D'Esposito M (2014) Revisiting the role of persistent neural activity during working memory. Trends Cogn Sci 18:8289. CrossRef Medline

Staudigl T, Hanslmayr S (2013) Theta oscillations at encoding mediate the context-dependent nature of human episodic memory. Curr Biol 23: 1101-1106. CrossRef Medline

Storey JD (2002) A direct approach to false discovery rates. J R Stat Soc Ser B 64:479-498. CrossRef

Tallon-Baudry C, Bertrand O, Peronnet F, Pernier J (1998) Induced gamma-band activity during the delay of a visual short-term memory task in humans. J Neurosci 18:4244-4254. Medline

Tallon-Baudry C, Bertrand O, Fischer C (2001) Oscillatory synchrony between human extrastriate areas during visual short-term memory maintenance. J Neurosci 21:RC177(1-5). Medline

Tort AB, Kramer MA, Thorn C, Gibson DJ, Kubota Y, Graybiel AM, Kopell N] (2008) Dynamic cross-frequency couplings of local field potential oscillations in rat striatum and hippocampus during performance of a T-maze task. Proc Natl Acad Sci U S A 105:20517-20522. CrossRef Medline

Tort AB, Komorowski R, Eichenbaum H, Kopell N (2010) Measuring phase-amplitude coupling between neuronal oscillations of different frequencies. J Neurophysiol 104:1195-1210. CrossRef Medline

Van Veen BD, van Drongelen W, Yuchtman M, Suzuki A (1997) Localization of brain electrical activity via linearly constrained minimum variance spatial filtering. IEEE Trans Biomed Eng 44:867-880. CrossRef Medline

von Nicolai C, Engler G, Sharott A, Engel AK, Moll CK, Siegel M (2014) Corticostriatal coordination through coherent phase-amplitude coupling. J Neurosci 34:5938-5948. CrossRef Medline

von Stein A, Sarnthein J (2000) Different frequencies for different scales of cortical integration: from local gamma to long range alpha/theta synchronization. Int J Psychophysiol 38:301-313. CrossRef Medline

Voytek B, Knight RT (2015) Dynamic network communication as a unifying neural basis for cognition, development, aging, and disease. Biol Psychiatry 77:1089-1097. CrossRef Medline

Woloszyn L, Sheinberg DL (2009) Neural dynamics in inferior temporal cortex during a visual working memory task. J Neurosci 29:5494-5507. CrossRef Medline 